\title{
Somatic responses in behavioral inhibition
}

\author{
Paul Whitney, John M. Hinson, Aaron Wirick, and Heather Holben \\ Washington State University, Pullman, Washington
}

\begin{abstract}
In the present study, skin conductance responses (SCRs) were measured postdecision and prefeedback in a go/no-go (GNG) task in which participants used response feedback to learn when to respond or not to respond to numeric stimuli. Like somatic markers in gambling tasks and somatic reactions to error monitoring in choice reaction time tasks, SCR patterns distinguished between correct and incorrect trials over time. These somatic reactions were disrupted by a reversal of GNG contingencies, and they were facilitated by pretraining of the stimulus-response mappings. In all cases, however, the somatic reactions appeared to be a product of competent decision making rather than a contributor to performance. Differential somatic responses to good and bad choices appear to be a robust and fairly general phenomenon, but researchers should be cautious in assuming that the somatic responses contribute to performance.
\end{abstract}

The ability to inhibit responses is an important component of cognitive control and a major focus of study in cognitive neuroscience. Failures of inhibitory control are characteristic of a wide range of neuropsychiatric disorders (see, e.g., Barkley, 1997; Hershey et al., 2004), and in the general population, inhibitory control problems are related to risk for substance abuse and other potentially damaging behavior problems (e.g., Finn, Justus, Mazas, \& Steinmetz, 1999; Swann, Bjork, Moeller, \& Dougherty, 2002). Research with brain-injured and normal subjects shows that the different tasks used to assess inhibitory control ability share some common processes, but no single mechanism or brain circuit controls all types of inhibition (cf. Hamilton \& Martin, 2005; Miyake et al., 2000). Even in the case of relatively simple motor inhibition tasks, there is a long-standing debate concerning whether the inhibition of initial motor activation is exercised by the central executive system or whether it is based on lateral inhibition without central executive control (see Band, Ridderinkhof, \& van der Molen, 2003; Gratton, Coles, Sirevaag, Eriksen, \& Donchin, 1988; Shimamura, 1995).

More recently, both neuroimaging and lesion data have indicated that the supplementary motor area, the dorsolateral prefrontal cortex (DLPFC), the anterior cingulate, and the ventromedial prefrontal cortex (VMPFC) are involved in response inhibition to varying degrees, depending on the response to be inhibited and the complexity of the task (see, e.g., Clark, Cools, \& Robbins, 2004; Dias, Robbins, \& Roberts, 1997; Mostofsky et al., 2003). One of the most well-validated procedures used in these studies of behavioral inhibition is the go/no-go (GNG) task (e.g., Band et al., 2003; Braver, Barch, Gray, Molfese, \& Snyder, 2001). The GNG task requires a rapid decision about whether to respond to a particular stimulus. For example, in a simple version of the GNG task, subjects are instructed to press a key when they see any letter other than $\mathrm{X}$, and to withhold a response to the letter X. Because the letter X appears on only about $20 \%$ of trials and the subject must respond rapidly, the prepotent response is to press the key. Problems with behavioral inhibition are assessed by the rate of keypresses to the letter X (i.e., false alarms). Although false alarms in GNG tasks are often considered to be a measure of motor impulsiveness, several different frontal circuits are involved in inhibiting the prepotent responses (e.g., Hershey et al., 2004; Mostofsky et al., 2003).

A somewhat more complex version of the GNG task that is relevant to the present research adds a learning component to the requirement of inhibiting a prepotent response (e.g., Finn et al., 1999; Newman \& Kosson, 1986). Subjects are presented two-digit numbers as stimuli and must learn which digits are in go and no-go sets on the basis of monetary gains and losses for correct and incorrect decisions. After the subjects learn which stimuli are in the go and no-go sets, the response requirements are reversed without warning so that the go stimuli become no-go stimuli, and vice versa. False alarms, particularly in the reversal phase, are an index of problems with behavioral inhibition. For example, Finn et al. found that alcohol administration increased false alarms in both the initial learning phase and the reversal phase of this GNG task. In the present study, we used this GNG task to investigate somatic responses associated with behavioral inhibition performance. Our aim was to determine whether participants would develop somatic reactions during the learning phase of the GNG task that are analogous to the reactions obtained in more deliberative decision tasks such as the Iowa gambling task (GT) (Bechara, 2004; Bechara, Damasio, Tranel, \& Damasio, 1997; Damasio, 1994).

On the basis of their research with the GT, Damasio, Bechara, and colleagues have proposed the somatic

P.Whitney, pwhitney@wsu.edu 
marker hypothesis, an influential theory of the role of somatic processes in decision making. In the GT, participants begin with a hypothetical stake of money and make choices from decks of cards that can increase or decrease their pool of money. The object of the GT is to make choices that will increase winnings as much as possible. As people make choices, their affective reactions are monitored using skin conductance responses (SCRs). In the most commonly used version of the GT, there are two "bad" decks that yield some large gains, but with even larger losses, and two "good" decks that yield smaller short-term gains, but which produce net long-term gains. Over time, the best performers make a higher proportion of choices from the good decks. As people learn to make more choices from the good decks, they also show anticipatory SCRs before making a choice. These SCRs, which Damasio and colleagues conceive of as somatic markers, allow one to distinguish between choices from good and bad decks. The somatic marker hypothesis claims that when a choice is made from alternatives that have each produced gains and losses, the VMPFC is responsible for activating neural circuitry that reconstitutes a somatic state. This somatic state, which can be monitored through SCRs, represents the integration of the previous instances of reward and punishment related to the choice options, and the activated somatic state can unconsciously guide the decision process (Bechara et al., 1997). The most compelling evidence that anticipatory SCRs may reflect a somatic process that facilitates decision making comes from studies of patients with damage to the VMPFC (e.g., Bechara et al., 1997). The VMPFC patients choose more cards from the bad decks, and, unlike normal controls, they do not show anticipatory SCRs to choices from good and bad decks.

The somatic marker hypothesis has become quite controversial, in part because of conflicting results over whether performance is determined by explicit knowledge of outcomes, and also because it has proven difficult to determine whether the anticipatory somatic reactions actually play a causal role in the decision making process (cf. Bechara, Damasio, Tranel, \& Damasio, 2005; Dunn, Dalgleish, \& Lawrence, 2005; Maia \& McClelland, 2004, 2005; Tomb, Hauser, Deldin, \& Caramazza, 2002). Nevertheless, there is an emerging consensus that the activity of the VMPFC is critical to reversal learning tasks in general, and the typical implementation of the GT is an example of a reversal learning task (Clark et al., 2004; Frank \& Claus, 2006; Rolls, 2004). It requires that the participants shift their choice strategy away from "bad" decks that initially produce good outcomes.

The GNG task used in the present study differs from the GT in many respects, particularly in the fact that the GT is based on deliberative choices on each trial, whereas the GNG task requires very rapid decision making. However, these tasks share a key feature. In both tasks, performance over trials depends on the ability to track reward contingencies and adjust them as needed. To illustrate how somatic reactions might play a role in the GNG task, consider the recent computational model of VMPFC func- tioning presented by Frank and Claus (2006). They modeled performance on both GNG and GT tasks in terms of the interactions among basal ganglia, VMPFC, and amygdala pathways. In their model, a key feature of both GT and GNG task performance is the top-down influence of recent reward associations maintained by the orbitofrontal cortex. On the basis of the Frank and Claus model as well as neuroimaging and lesion data showing that the VMPFC is critical to performance in paradigms that involve tracking and updating changes in the reward or emotional significance of stimuli (e.g., Rolls, 2004), one would expect that the differential reward magnitudes associated with the various choice options could generate a somatic reaction in a wide range of paradigms.

What is less clear are the conditions in which somatic reactions influence task performance. For example, as the contingencies are learned in the GNG task, there may be somatic reactions associated with making a particular response. When contingencies are reversed, the mismatch between the affective reaction associated with making a response and the affective reaction to the actual feedback obtained could support the ability to adapt to the reversal. We know that in reversal learning tasks there are ERP and SCR indices that are specific to obtaining a reward outcome that is larger or smaller than expected (see, e.g., Crone, Vendel, \& van der Molen, 2003; Hajcak, McDonald, \& Simons, 2004). The expectation that drives these effects could have an affective component that looks much like the somatic markers obtained with the GT. Alternatively, somatic reactions might develop in response to expectations about the likely outcome of a decision, but the somatic effect could be epiphenomenal and play no causal role in performance. In the latter case, the somatic effects would simply be a by-product of the expectation processing.

In the present study, participants performed a GNG task based on the procedures used by Finn et al. (1999) and Newman and Kosson (1986). The subjects had to learn which digits were in the go and no-go sets, but we extended the time period between the response deadline and the feedback to the participant in order to measure SCRs during the period in which the participants could be expected to anticipate the outcomes of their decisions. Our primary interest in the first experiment was whether improvements in decision accuracy over trials would be accompanied by differential SCR patterns in anticipation of correct and incorrect responses. The somatic effects of interest should be manifested as differential SCR responses on correct and incorrect trials before accuracy feedback is given. This outcome would show that a somatic response in a GNG task could, in principle, be used to adjust performance over trials.

\section{EXPERIMENT 1}

\section{Method}

Participants. Eighty-four students from the introductory psychology course at Washington State University participated in the study. They received credit in partial fulfillment of a research par- 
ticipation requirement. The sample was approximately $60 \%$ female, and the students ranged in age from 18 to 25 years.

Procedure. Figure 1 shows the timing of events used for the GNG task. Each trial began with a 4-sec preparatory period to allow SCRs from the previous trial to return to baseline. There was a countdown during the preparatory period in which a string of asterisks appeared, one at a time, at 1-sec intervals. At the end of the countdown, a two-digit number was presented, and the participant had $750 \mathrm{msec}$ to respond. After the response deadline, the person was informed whether or not a response was detected, to remove any ambiguity between a nonresponse and a response made after the deadline. However, no accuracy feedback was given until $2.5 \mathrm{sec}$ later. In that 2.5 -sec interval, we measured changes in SCR.

The general procedure, including the fact that the task would involve hypothetical gains and losses of money, was explained to the participants orally by the experimenter. Then the participants were told to read the specific instructions for the task on the computer screen, which were as follows:

In this experiment you will be asked to respond or withhold a response in order to win money. You will be given an initial stake of $\$ 5$. After that, every few seconds a number will be presented. On each occasion you must decide whether to respond by pressing the space bar or withhold a response by not pressing the space bar on that occasion.

To assist you in making a speeded choice, you will be told whether or not you have made a valid response on each trial. If you press the space bar within the time limit the computer will display “RESPONSE." If you wait too long, or make no response on the trial, the computer will display "NO RESPONSE."

Following this, you will be told whether your RESPONSE or NO RESPONSE on the trial was correct or incorrect.

If your choice is correct, you will win \$.20.

If your choice is incorrect, you will lose $\$ .20$.

Please note that you will have only a limited amount of time to choose to respond. If you wait too long, we will record that as withholding a response.

The participants were seated in front of individual computer terminals and told to begin when ready. A program written in Microsoft Visual Basic provided the written instructions and collected all of the data from the experiment. As described below, the left hand was used for SCR recording, while the right hand rested on the space bar of a standard keyboard. Stimuli were presented in the center of the computer screen, which was about 18 in. in front of the participant. Two sets of four two-digit numbers were used as stimuli. Set 1 contained 58, 19, 14, and 79. Set 2 contained 28, 63, 74, and 25. The use of Set 1 and Set 2 numbers as either go or no-go stimuli was

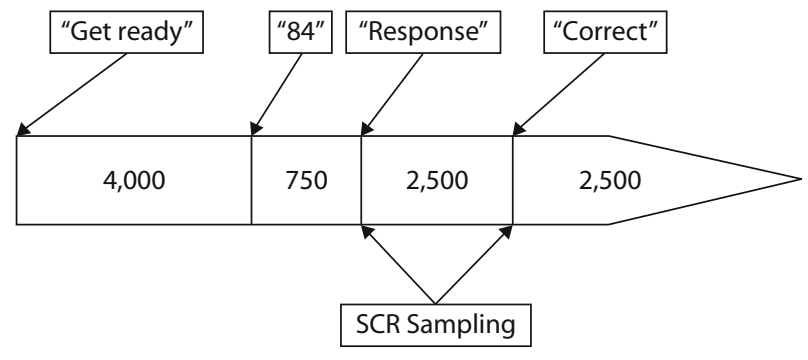

Trial Timeline (msec)

Figure 1. Timing of the trial events in the go/no-go task. The SCR sampling isolates the phase of the trial in which the participant has made the choice to respond or not but has not yet received accuracy feedback.

counterbalanced between participants. Participants received 80 total trials. The sequence of stimuli was randomized across these 80 trials, with the constraint that within a block of 8 trials, there would be no more than three consecutive presentations of members of the go or no-go stimulus sets.

Skin conductance response measurement. SCR was recorded by means of a Contact Precision Instruments SC5 SA skin conductance monitor. For analysis of somatic responsiveness, we measured changes in SCR amplitude (see, e.g., Dawson, Schell, \& Filion, 2000; Hinson, Jameson, \& Whitney, 2002). Beginning with a response or when the time deadline for the response had expired, SCL was sampled to establish the baseline level for that trial. SCL was then continuously sampled until the trial outcome feedback was provided. The SCR amplitude for the trial was calculated as the difference between peak SCL and baseline SCL on that trial. Any difference between peak SCL and baseline SCL of less than $.01 \mu \mathrm{S}$ was considered to be no SCR on that trial. Further details of the recording procedure may be found in Hinson et al.

\section{Results and Discussion}

The decision data and SCR data are summarized in Figure 2. Not surprisingly, performance improved over blocks of trials (organized for clarity as five blocks of 16 trials each). Repeated measures ANOVA confirmed that false alarms decreased over blocks $[F(4,332)=21.17, p<$ $\left..001, \eta^{2}=.20\right]$ and hits increased over blocks $[F(4,332)=$ $\left.13.89, p<.001, \eta^{2}=.14\right]$.

Of greater interest were the SCR data, which were analyzed in a $2 \times 5$ (response type $\times$ block) repeated mea-
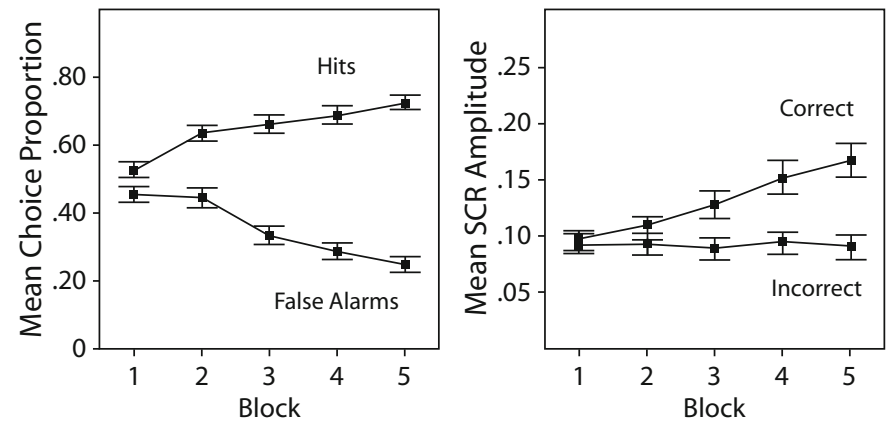

Figure 2. Data from Experiment 1. The left panel depicts means (and standard errors) of hits and false alarms by trial block for the decision data. The right panel depicts the SCR amplitudes (measured in $\mu \mathrm{S}$ ) for each block as a function of decision accuracy 
sures ANOVA. The response type variable refers to whether the SCR was from a trial on which the choice was correct or incorrect. The main question of interest was whether, over blocks, a difference in SCRs would emerge between correct and incorrect trials. A necessary condition for somatic responses to influence choice behavior over trials is that the SCR pattern must distinguish between correct and incorrect choices (see Hajcak, McDonald, \& Simons, 2003; Hinson et al., 2002; Jameson, Hinson, \& Whitney, 2004). We did obtain such a pattern. There were significant main effects of response type $[F(1,83)=29.92, p<$ $.001]$ and block $[F(4,332)=7.46, p=.011]$, and a significant interaction of the two variables $[F(4,332)=9.79$, $p<.001]$. A breakdown of this interaction is provided in Table 1, which summarizes the contrasts of correct versus incorrect response type at each block. ${ }^{1}$ The data show that reliable somatic reactions were obtained on the GNG task, and by the end of the task the differential response to correct and incorrect trials was quite substantial in terms of the effect size.

Although we obtained differential SCRs on correct and incorrect trials, the data do not indicate that the somatic reactions played a causal role in performance improvements over trials. The differential somatic reactions in the GNG task were a lagging indicator, rather than a leading indicator, of performance. Even in Block 1, there was a significant difference in hit and false alarms rates $[F(1,83)=$ $\left.5.98, p=.017, \eta^{2}=.07\right]$, but it clearly took longer for the differential SCR response to develop. In the GNG task, the somatic reactions appear to have represented an effect of the cognitive processes that produced good performance. It seems most likely to us that we observed a somatic reaction to the anticipation of a reward. That is, in the GNG task, the person made a choice to respond or not to respond, and the somatic reaction developed during a phase of the task in which the person's performance was competent enough for the person to correctly anticipate whether the choice would lead to positive or negative outcomes. A somewhat different potential source for the SCR elevation on correct trials would be a somatic reaction to knowing that a response was correct. That is, it might not be the anticipation of reward, but rather an affective response to producing a correct response that we were observing. It was not possible to distinguish between these possibilities in this design, and it may be very difficult to distinguish between them in principle because they might rely on the same reward circuitry.

Another potential interpretation of the SCR effects observed here is that they represented a somatic index of

Table 1

Contrasts of SCR Amplitudes on Correct Versus Incorrect Trials at Each Block in Experiment 1

\begin{tabular}{cccc}
\hline Block & $F(1,83)$ & $p$ & $\eta^{2}$ \\
\hline 1 & $<1$ & - & - \\
2 & 3.11 & .08 & .04 \\
3 & 12.27 & .001 & .13 \\
4 & 21.06 & $<.001$ & .20 \\
5 & 25.62 & $<.001$ & .24 \\
\hline
\end{tabular}

error monitoring. In a two-choice reaction time task, Hajcak et al. (2003, 2004; see also Crone, Somsen, van Beek, \& van der Molen, 2004) obtained differential SCRs on correct and incorrect trials, and the effects emerged within $2.5 \mathrm{sec}$ of making a response, just as we found in the present study. Hajcak et al. (2003, 2004) attributed these somatic effects to error monitoring and suggested that they might be a "somatic marker of erring" (Hajcak et al., 2003 , p. 901). It seems unlikely, however, that the present results were based on the same SCR index as the one identified by Hajcak and colleagues. Hajcak et al. (2003, 2004) found higher SCRs on error trials than on correct trials. The present data showed the opposite pattern.

Whatever the exact source of the somatic effect, we were able to obtain differential somatic reactions to good and bad choices in the GNG task. However, the differential SCR patterns appear to have been produced by competent task performance as opposed to facilitating performance. We suggest that the somatic effect emerged only after the person had learned the go and no-go sets well enough that they would know whether they had made a correct response and could anticipate the feedback they would receive after making a response. To further test this idea, we replicated our first experiment, but with a training phase so that participants would not have to learn the go and no-go sets from feedback on their decisions.

\section{EXPERIMENT 2}

Because the somatic effects in the first experiment were obtained postresponse and prefeedback, and because the differential SCRs consistently lagged behind competent performance, we interpreted these results as indicative of a somatic reaction to an anticipated outcome rather than an influence on the decision processes over trials. If our interpretation was correct, then as long as the participant could confidently anticipate the outcome of a trial, we should be able to obtain the differential somatic reactions observed in the first experiment. In the second experiment, the participants were trained on which numbers were in the go and no-go sets before the GNG task began, and we predicted that the somatic effects would emerge immediately.

\section{Method}

Participants. Thirty-five students from the same pool as that for the previous experiment were recruited. The composition of the sample was very similar to that in the previous experiment.

Procedure. With the exception of the training period and the specific stimulus numbers used, the procedural details of Experiment 2 were the same as those in Experiment 1. In Experiment 2, Set 1 contained 38, 11, 97, and 62, whereas Set 2 contained 86, 17, 61, and 42 . The assignment of Set 1 and Set 2 numbers as go or no-go stimuli was counterbalanced between participants. The participants were provided with training on the go and no-go stimulus sets before beginning the GNG task. Each person was told to read the instructions for the training task, which appeared on the computer screen as follows:

We would like you to learn two sets of numbers before you begin the next part of the experiment. For one set of numbers you will later be asked to make a response on the Space Bar. 
For the other set of numbers, you will be asked to withhold your response.

The participants were next given a listing of the numbers and the associated go or no-go response that would be required in the next task. Then they were asked to memorize the numbers. After a few seconds, the participant was asked to enter the go and no-go sets. If there were errors, the computer displayed the numbers to allow proper memorization. The process was continued until the participant had correctly identified all numbers on three consecutive attempts. After memorizing the stimulus sets, the participants were given the instructions described in Experiment 1, followed by 80 GNG trials.

\section{Results and Discussion}

The decision data and SCR data are summarized in Figure 3. As in the previous experiment, there was an increase in hit rates over trials $[F(4,136)=12.6, p<.001$, $\left.\eta^{2}=.27\right]$, but unlike in the other experiments, false alarm rates were low from the beginning and stayed flat over trial blocks $(F<1)$. So, although there was some improvement in performance over trials, it is clear that the training was effective and performance was competent from the beginning. For the SCR data, the response type $\times$ block repeated measures ANOVA showed that SCRs on correct trials were higher than SCRs on incorrect trials $\left[F(1,34)=22.36, p<.001, \eta^{2}=.39\right]$. The effect of block was marginal $\left[F(4,136)=1.87, p=.11, \eta^{2}=.05\right]$, and there was no interaction $(F<1)$. Thus, as predicted, training allowed the participants to perform well from the start, and differential SCRs were immediately evident. It appears that tracking of response contingencies over trials is not required to obtain the somatic effects observed in Experiment 1. A minimal condition sufficient to obtain differential SCRs in the GNG task is that the person can competently distinguish between go and no-go stimuli.

\section{EXPERIMENT 3}

In Experiment 3, we implemented the GNG task with the typical reversal phase that is most often used to study problems with behavioral inhibition. We introduced a reversal of go and no-go sets after four blocks of 16 trials.
We expected false alarms to increase immediately after the reversal and to recover quickly. On the basis of the first two experiments, the differential SCRs should be disrupted by the reversal at the same time that performance decreased. Our primary question was whether changes in the SCRs would be a leading or lagging indicator of performance after the reversal.

\section{Method}

Participants. A new sample of 40 students from the same pool as that for the previous experiment was recruited. The composition of the sample was very similar to that in the previous experiments.

Procedure. As in the previous experiments, two sets of stimuli were employed with four two-digit numbers in each set. Set 1 contained 38,11,97, and 62, whereas Set 2 contained 86, 17, 61, and 42. The assignment of Set 1 and Set 2 numbers as go or no-go stimuli was counterbalanced between participants. Each participant received 104 total trials. After 64 trials, the assignment of go and no-go stimuli was reversed. This reversal of go and no-go stimuli continued for the 40 remaining trials.

\section{Results and Discussion}

In order to track the trends of interest to the hypotheses, the trials were divided into four blocks of 16 trials in the prereversal phase, then a block of 8 trials in which we could observe the acute effects of the reversal, followed by two more blocks of 16 trials in which we could examine the recovery of performance. The decision and SCR data are summarized in Figure 4.

The analysis of hits closely mirrored that of false alarms, so we present only the false alarm analyses here. The number of false alarms varied significantly by block $\left[F(6,234)=12.93, p<.001, \eta^{2}=.25\right]$. Within this main effect, we examined the effect of reversal, as well as recovery from it, with a set of planned contrasts that compared the number of false alarms in the reversal block with the number in the immediately preceding block and with false alarms in the two postreversal blocks. When the reversal occurred, there was a significant and substantial increase in false alarms, but performance recovered very rapidly with significant reductions in false alarms in the next block of trials that returned performance to its prereversal level. These analyses are summarized in Table 2.
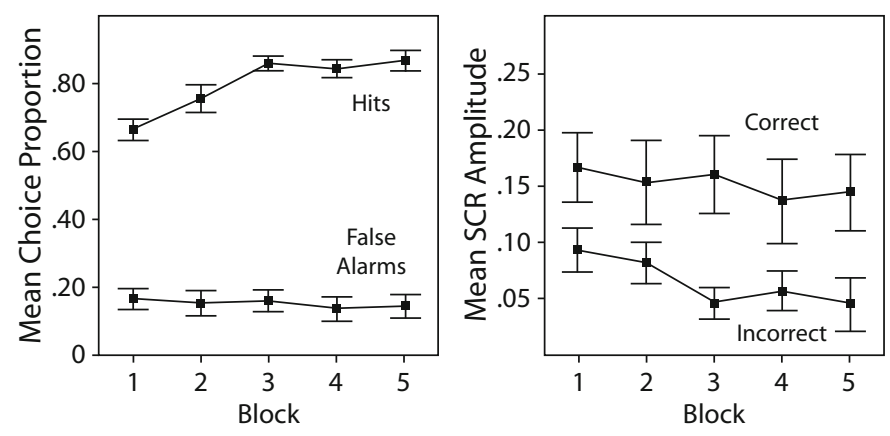

Figure 3. Data from Experiment 2, which replicated the first experiment except that participants received training on identifying go and no-go stimuli before the trials began. The left panel depicts the decision data, and the right panel depicts the SCR amplitudes as a function of decision accuracy. 

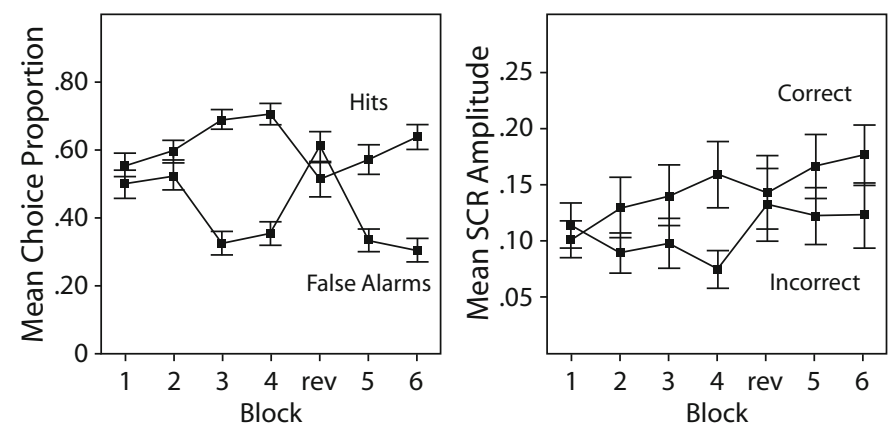

Figure 4. Data from Experiment 3, in which a reversal of the go and no-go stimuli was implemented. The reversal block (rev) consisted of the 8 trials immediately after the reversal. The other blocks consisted of 16 trials as in Experiments 1 and 2. The left panel shows the decision data, and the right panel shows the SCR amplitudes by decision accuracy.

The SCR data were analyzed in a $2 \times 7$ (response type $\times$ block) repeated measures ANOVA. SCR amplitudes were higher on trials in which the decision was correct rather than incorrect $[F(1,39)=13.15, p=.001]$. There was no significant main effect of block $(p=.27)$, but the response type $\times$ block interaction was significant $[F(6,234)=2.36, p=.04]$. As Figure 4 indicates, a significant pattern of differential SCRs was established by Block $2[t(39)=2.057, p=.04]$, and it continued for the next two blocks $[t(39)=2.28, p=.03 ; t(39)=3.40, p=$ .002 , respectively]. The pattern disappeared in the reversal block $(p=.55)$ and was reestablished in Block $6[t(39)=$ $2.37, p=.02]$, but not in Block $5[t(39)=1.64, p=.11]$, when decision performance had already returned to near prereversal levels. Consistent with the interpretation that the somatic reactions did not play a causal role in performance, the somatic effects were a lagging indicator of behavioral inhibition in the postreversal phase.

\section{GENERAL DISCUSSION}

Across three experiments, we obtained differential SCRs to correct and incorrect trials after decision performance had become competent. The results of this study contribute to the growing body of evidence that reactions in the autonomic nervous system are associated with several kinds of decision tasks. In the prior cases of deliberative decisions in simulated gambling and error monitoring in choice RT, it has been unclear whether the somatic reactions actually contribute to performance. In the somatic reactions we have documented in the GNG, the case seems more clear. The SCR data in this task are

Table 2

Contrasts of False Alarms in the Reversal Block With Preceding and Subsequent Blocks

\begin{tabular}{cccc}
\hline Contrast & $F(1,39)$ & $p$ & $\eta^{2}$ \\
\hline Reversal vs. Block 4 & 34.96 & $<.001$ & .47 \\
Reversal vs. Block 5 & 28.27 & $<.001$ & .42 \\
Reversal vs. Block 6 & 48.96 & $<.001$ & .56 \\
\hline
\end{tabular}

produced by good choice behavior; they do not produce such behavior. Somatic reactions may play a causal role in other decision tasks, but our data add to a growing chorus of caution about assuming that somatic reactions are contributors to decision making, rather than a consequence of having made a decision (cf. Fellows \& Farah, 2003; Maia \& McClelland, 2005).

In considering the implications of the present results for future studies, it is interesting to consider how the GNG task and other reversal learning tasks, including the GT, relate to real-world decision making. For example, suboptimal performance on both the GNG task and the GT have been interpreted in terms of impulsive decision making, and poor performance on both of these tasks has been related to the similar overt manifestations of impulsiveness, including risk for substance abuse (Bechara \& Damasio, 2002; Clark et al., 2004; Ernst et al., 2003; LeMarquand, Benkelfat, Pihl, Palmour, \& Young, 1999). Bechara et al. (2005) suggested that the somatic marker may serve as a signal that is used to inhibit behavior in reversal learning paradigms. However, the kinds of reversal learning paradigms to which they refer involve deliberation over choices that are more similar to those in the GT paradigm than in the GNG task (cf. Fellows \& Farah, 2003). An important avenue to explore for future research is whether deliberative tasks that involve inhibition tap a different form of impulsiveness than do more speeded tasks such as the GNG. If so, it is possible that the difference in types of impulsiveness assessed by different decision tasks is related to differences in whether or not somatic reactions contribute to the decision process.

\section{AUTHOR NOTE}

Portions of these data were presented at the 2005 annual meeting of the Cognitive Neuroscience Society. Correspondence should be addressed to P. Whitney, Department of Psychology, P.O. Box 644820, Washington State University, Pullman, WA 99164-4820 (e-mail: pwhitney@wsu.edu).

\section{REFERENCES}

Band, G. P. H., Ridderinkhof, K. R., \& van der Molen, M. W. (2003). Speed-accuracy modulation in case of conflict: The roles of activation and inhibition. Psychological Research, 67, 266-279. 
BARKLEY, R. A. (1997). Behavioral inhibition, sustained attention, and executive functions: Constructing a unifying theory of ADHD. Psychological Bulletin, 121, 65-94.

BECHARA, A. (2004). The role of emotion in decision-making: Evidence from neurological patients with orbitofrontal damage. Brain \& Cognition, 55, 30-40.

Bechara, A., \& Damasio, H. (2002). Decision making and addiction (part I): Impaired activation of somatic states in substance dependent individuals when pondering decisions with negative future consequences. Neuropsychologia, 40, 1675-1689.

Bechara, A., Damasio, H., Tranel, D., \& Damasio, A. R. (1997). Deciding advantageously before knowing the advantageous strategy. Science, 275, 1293-1294.

Bechara, A., Damasio, H., Tranel, D., \& Damasio, A. R. (2005). The Iowa Gambling Task and the somatic marker hypothesis: Some questions and answers. Trends in Cognitive Neurosciences, 9, 159-162.

Braver, T. S., Barch, D. M., Gray, J. R., Molfese, D. L., \& Snyder, A. (2001). Anterior cingulate cortex and response conflict: Effects of frequency, inhibition and errors. Cerebral Cortex, 11, 825-836.

Clark, L., Cools, R., \& Robbins, T. R. (2004). The neuropsychology of ventral prefrontal cortex: Decision-making and reversal learning. Brain \& Cognition, 55, 41-53.

Crone, E. A., Somsen, R. J. M., van Beek, B., \& van der Molen, M. W. (2004). Heart rate and skin conductance analysis of antecedents and consequences of decision making. Psychophysiology, 41, 531-540.

Crone, E. A, Vendel, I., \& van der Molen, M. W. (2003). Decision making in disinhibited adolescents and adults: Insensitivity to future consequences or driven by immediate reward? Personality \& Individual Differences, 35, 1625-1641.

Damasio, A. R. (1994). Descartes' error: Emotion, reason and the human brain. New York: Grosset/Putnam.

Dawson, M. E., Schell, A. M., \& Filion, D. L. (2000). Handbook of psychophysiology. Cambridge: Cambridge University Press.

Dias, R., Robbins, T. W., \& RoberTs, A. C. (1997). Dissociable forms of inhibitory control within prefrontal cortex with an analog of the Wisconsin card sort test: Restriction to novel situations and independence from "on-line" processing. Journal of Neuroscience, 17, 9285-9297.

Dunn, B. D., Dalgleish, T., \& Lawrence, A. D. (2005). The somatic marker hypothesis: A critical evaluation. Neuroscience \& Biobehavioral Reviews, 30, 239-271.

Ernst, M., Grant, S. J., London, E. D., Contoreggi, C. S., Kimes, A. S., \& Spurgeon, L. (2003). Decision making in adolescents with behavior disorders and adults with substance abuse. American Journal of Psychiatry, 160, 33-42.

Fellows, L. K., \& Farah, M. J. (2003). Ventromedial frontal cortex mediates affective shifting in humans: Evidence from a reversal learning paradigm. Brain, 126, 1830-1837.

Finn, P. R., Justus, A., Mazas, C., \& Steinmetz, J. E. (1999). Working memory, executive processes and the effects of alcohol on go/no-go learning: Testing a model of behavioral regulation and impulsivity. Psychopharmacology, 146, 465-472.

Frank, M. J., \& Claus, E. D. (2006). Anatomy of a decision: Striatoorbitofrontal interactions in reinforcement learning, decision making and reversal. Psychological Review, 113, 300-326.

Gratton, G., Coles, M. G., Sirevaag, E. J., Eriksen, C. W., \& Donchin, E. (1988). Pre- and poststimulus activation of response channels: A psychophysiological analysis. Journal of Experimental Psychology: Human Perception \& Performance, 14, 331-344.

Hajcak, G., McDonald, N., \& Simons, R. F. (2003). To err is autonomic: Error-related brain potentials, ANS activity, and post-error compensatory behavior. Psychophysiology, 40, 895-903.

Hajcak, G., McDonald, N., \& Simons, R. F. (2004). Error-related psychophysiology and negative affect. Brain \& Cognition, 56, 189-197.

Hamilton, A. C., \& Martin, R. C. (2005). Dissociations among tasks involving inhibition: A single-case study. Cognitive, Affective, \& Behavioral Neuroscience, 5, 1-13.

Hershey, T., Black, K. J., Hartlein, J., Braver, T. S., Barch, D. M.,
CARL, J. L., ET AL. (2004). Dopaminergic modulation of response inhibition: An fMRI study. Cognitive Brain Research, 20, 438-448.

Hinson, J. M., Jameson, T. L., \& Whitney, P. (2002). Somatic markers, working memory, and decision making. Cognitive, Affective, \& Behavioral Neuroscience, 2, 341-353.

Jameson, T. L., Hinson, J. M., \& Whitney, P. (2004). Components of working memory and somatic markers in decision making. Psychonomic Bulletin \& Review, 11, 515-520.

LeMarquand, D. G., Benkelfat, C., Pihl, R. O., Palmour, R. M., \& YounG, S. N. (1999). Behavioral disinhibition induced by tryptophan depletion in nonalcoholic young men with multigenerational family histories of paternal alcoholism. American Journal of Psychiatry, 156, 1771-1779.

Maia, T. V., \& McClelland, J. L. (2004). A reexamination of the evidence for the somatic marker hypothesis: What participants really know in the Iowa gambling task. Proceedings of the National Academy of Sciences, 101, 16075-16080.

Maia, T. V., \& McClelland, J. L. (2005). The somatic marker hypothesis: Still many questions but no answers. Trends in Cognitive Sciences, 9, 162-164.

Miyake, A., Friedman, N. P., Emerson, M. J., Witzki, A. H., Howerter, A., \& WAGER, T. D. (2000). The unity and diversity of executive functions and their contributions to complex "frontal lobe" tasks: A latent variable analysis. Cognitive Psychology, 41, 49-100.

Mostofsky, S. H., Schafer, J. G. B., Abrams, M. T., Goldberg, M. C., Flower, A. A., BoyCE, A., ET AL. (2003). fMRI evidence that the neural basis of response inhibition is task-dependent. Cognitive Brain Research, 17, 419-430.

Newman, J. P., \& Kosson, D. S. (1986). Passive avoidance learning in psychopathic and nonpsychopathic offenders. Journal of Abnormal Psychology, 95, 252-256.

RoLLS, E. T. (2004). The functions of the orbitofrontal cortex. Brain \& Cognition, 55, 11-29.

Shimamura, A. P. (1995). Memory and frontal lobe function. In M. S. Gazzaniga (Ed.), The cognitive neurosciences (pp. 803-813). Cambridge, MA: MIT Press.

Swann, A. C., Bjork, J. M., Moeller, F. G., \& Dougherty, D. M. (2002). Two models of impulsivity: Relationship to personality traits and psychopathology. Biological Psychiatry, 51, 988-994.

Tomb, I., Hauser, M., Deldin, P., \& Caramazza, A. (2002). Do somatic markers mediate decisions on the gambling task? Nature Neuroscience, 5, 1103-1104.

Whitney, P., JAmeson, T., \& Hinson, J. M. (2004). Impulsiveness and executive control of working memory. Personality \& Individual Differences, 37, 417-428.

\section{NOTE}

1. There are several reasons for analyzing the SCR data according to correct and incorrect trials, the primary one being that if somatic reactions guide performance improvements over trials, they should allow one to distinguish between correct and incorrect choices. Analyzing the data in this way makes the results more comparable to analyses of somatic reactions in other choice tasks (cf. Bechara et al., 1997; Hajcak, McDonald, \& Simons, 2004). In addition, SCRs are rather noisy signals, so it is important to average over as many trials as possible in order to have sufficient power to detect differential somatic reactions. Nevertheless, to ensure that averaging over correct and incorrect trials was not obscuring other important trends in the data, we conducted one-way repeated measures ANOVAs on the block effect for each type of choice. For both hits and correct rejections, there was an increase in SCR amplitude over blocks $[F(4,332)=11.72, p<.001$, and $F(4,32)=6.89, p<$ .001 , respectively]. For both false alarms and misses, the SCR profile was statistically flat over blocks (both $p \mathrm{~s}>.20$ ). Therefore, the means depicted in Figure 2 are accurate representations of each type of correct and incorrect response.

(Manuscript received August 25, 2005; revision accepted for publication October 5, 2006.) 